Burkitt, E. \& Barrett, M. (2011). The effects of different drawing materials on children's drawings of positive and negative human figures. Educational Psychology, 31, 459-479.

\title{
The effects of different drawing materials on children's drawings of positive and negative human figures
}

\begin{abstract}
Children tend to use certain drawing strategies differentially when asked to draw topics with positive and negative emotional characterisations. These effects have however only been established when children are asked to use standard drawing materials. The present study was designed to investigate whether the above pattern of children's response when drawing characterised figures would alter when children are asked to use different drawing materials. 132 children (69 boys and 63 girls) aged between 4 and 11 years were divided into two conditions and completed two counterbalanced test sessions, rating colour preferences and drawing characterised figures using either stick or block crayons. It was found that some drawing strategies varied in relation to drawing materials and in relation to the precise characterisation employed. The results are discussed in terms of the need for interpretations of emotional information in children's drawings to take account of the exact materials used.
\end{abstract}




\section{The effects of different drawing materials on children's drawings of positive and negative human figures}

It has been a longstanding view that the features present in children's drawings may reflect more than the cognitive and motor skills involved in the drawing process (e.g. Aronsson \& Andersson, 1996; Cleeve \& Bradbury, 1992; Hammer, 1953, 1997; Kopptiz, 1968; Machover, 1949; Thomas \& Jolley, 1998; Thomas \& Silk, 1990). Departing from research traditions which incorporate psychodynamic interpretations of children's drawings, for example by claiming that children's personality or mood is projected onto the figures that they draw (e.g. Hammer, 1953, 1997; Kopptiz, 1968; Machover, 1949), recent research has taken an experimental approach to ascertain whether specific properties of drawings can be interpreted as indications of information about how the child artist feels towards the topics they have drawn (e.g. Cleeve \& Bradbury, 1992; Jolley, 1995; Thomas, Chaigne \& Fox, 1989). Research within this perspective has shown that when children are asked to copy or draw nice and nasty figures, or positive and negative men, they tend to produce larger positively characterised figures than baseline figures and draw positively salient figures larger than negatively characterised figures (e.g. Aronsson \& Andersson, 1996; Burkitt \& Barnett, 2006; Burkitt, Barrett \& Davis, 2003a, 2004; Fox \& Thomas, 1990). Children also choose colours which are related to their colour preferences to complete the drawings, and use a wide range of additional strategies to differentiate the emotional character of the drawn figures (Burkitt \& Barrett, 2010; Burkitt, Barrett \& Davis, 2003b, 2004; Nelson, Allan \& Nelson, 1971).

These effects have been established when children were given the standard drawing materials of lead pencils and stick crayons. Research examining children's 
representations of emotional information has tended to provide children with a wide range of drawing and painting equipment, for example paints, crayons, lead pencils or a combination of media (e.g. Alschuler \& Hattwick, 1943, 1947; Burkitt et al., 2004; Nelson, Allan \& Nelson, 1971) yet the subsequent claims are not qualified in light of the demands of specific materials on the resultant drawing (e.g. Burkitt et al., 2004; Callaghan, 1999; Cleeve \& Bradbury, 1992; Thomas, Chaigne \& Fox, 1989).

However, it is clear that children may draw a figure smaller or larger simply as a result of whether they are using a pencil or paint set rather than the size changes necessarily indicating a specific feeling of the child towards the drawn figure. Given that the properties of children's drawings continue to be used in interviews and assessments for information about the thoughts and feelings of children (e.g. Hammer, 1997; Liebowitz, 1999; Stefanatou \& Bowler, 1999), it is important that further understanding of the factors that shape children's drawings be understood in order for more accurate interpretations of children's drawings to be reached. Children's drawings are cue-dependent (Freeman, 1988, 1995), meaning that the exact inter- and intradrawing cues present in the drawing situation intimately shape the final drawing. Whilst we know that task instructions (e.g. Barrett, Beaumont \& Jennings, 1985), page orientation and preceding drawn lines (e.g. Freeman, 1988) influence the properties of a resultant drawing, less is known about the extent to which drawing materials affect content and, more importantly, whether the content is due to the drawing materials used or how the child artist feels towards the figures they draw. However, it may be very important to know whether a child has used pencils, crayons or paints when interpreting his or her drawings for emotional information and expressiveness.

Children's graphic flexibility when depicting factual information in response to a wide range of cues within drawings tasks is well established (e.g. Barrett \& Bridson, 
1983; Callaghan, 1999; Freeman, 1985; Light \& Simmons, 1983). However, the impact of the cues associated with specific task materials on the finished drawing has been under-explored within the literature, especially with tasks where the encoding of emotional information is involved. The present study was therefore designed to investigate whether children's representations of emotionally described humans, which they feel positively or negatively about, would vary as a function of a manipulation of the drawing materials provided.

The effects of drawing materials

The role of drawing materials has been previously investigated in relation to an ongoing debate about the nature of the relationship between representation and cognition. From the classical conceptual perspective (Goodenough, 1926; Luquet, 1913, 1927; Piaget \& Inhelder, 1956, 1969), a close correspondence between reasoning and representation exists, so that young children's drawings are reflections of their concepts of the drawn objects. This view predicts a high degree of uniformity across drawing conditions, which is largely unaffected by task media and instructions. In contrast to the conceptual theory, Arnheim (1956) describes the process of representation as the creation of structural equivalents in a specified medium. This position thus predicts that drawing performance will vary across drawing tasks as a function of the given medium and instructions.

The evidence suggests that the latter account is more accurate. For example, research examining the progression from intellectual to visual realism suggests that task demands can greatly influence the nature of children's representations (Arrowsmith, Cox \& Eames, 1994; Barrett \& Bridson, 1983; Barrett \& Light, 1976; Barrett, Beaumont \& Jennett, 1985; Barrett, Sutherland \& Lee, 1987; Chen \& Holman, 1984; Cox, 1981, 1985; Cox \& Parkin, 1986; Davis, 1983, 1985a, 1985b; Davis \& Bentley, 
1984; Lewis et. al., 1993; Light \& McEwan, 1987; Light \& Simmons, 1983; Parsons, 1995; Sitton \& Light, 1992). Golomb's (1973) classic study aiming to directly test these opposing theories through a series of representational tasks with 3 to 7 year olds, where the level of instructions and type of media were systematically varied, indicated that representation varies in accordance with the medium involved, the level of task instructions, the provision of parts, practice, and children's general developmental level. In terms of the cue-dependency framework proposed to explain variations in children's drawings as a result of the various cues within the drawing itself and the broader drawing context (Freeman, 1985), the precise materials used in production arguably constitute an integral cue which impacts on the drawn product.

Golomb also found that the differences in approach and performance across the range of representational tasks improved with age. For example, older children modelled, copied and drew figures with a similar style of construction and degree of completion more cohesively than the younger children. Golomb concluded that "each medium has its own laws and demands of articulation" (p. 247), and that young children are not entirely limited by their conceptual immaturity when approaching a drawing task.

Conflicting findings come from Brittain and Chien (1980), who claimed that materials only play a minor role in human figure representation. Brittain's (1986) study aimed to address this empirical discrepancy, and was also designed to examine the differences in procedures between Golomb's (1973) work and his own (1980). He tested nursery school children on a range of representational tasks, including drawing tasks, paper assembly tasks, and a wooden puzzle assembly task utilised by Golomb (1973). The main findings offered support of both perspectives. Success on the drawing task differed from successful performance on the other tasks, yet there were no 
significant differences in performance on the puzzle tasks. Whilst children showed mixed success across the tasks, he found that the representation of the human figure across the tasks was significantly correlated, namely a low scoring child on the drawing task obtained a low score on the puzzle tasks. The discrepancy (Brittain \& Chien, 1980; Golomb, 1973) thus appears to lie in the interpretation of the results rather than the empirical findings, largely arising, in Brittain's view, from Golomb's not correlating children's performance between tasks, instead accepting absolute scores from each task separately. Brittain maintains that whilst differences in representational media may play a role in children's performance on representational tasks, children's developmental level remains relatively constant across tasks. This position is also supported by research across a range of drawing tasks which suggests that although there are individual differences in children's human figure drawings, children tend to pass through general developmental phases (e.g. Barrett \& Eames, 1996; Cox, 1992, 1993; Cox \& Parkin, 1986; Fenson, 1985; Goodnow, 1977).

In the present study, a more specific manipulation of materials was made than those used in the studies by Golomb (1973) and Brittain (1986), in order to explore more specifically whether a change in drawing materials would influence children's depiction of emotional information in their drawings of positive and negative men, and whether any effects of the drawing materials would vary according to children's age.

The standard drawing materials employed in previous research (e.g. Burkitt et al., 2003b, 2004; Burkitt \& Newell, 2005), namely Crayola stick crayons, were given to one group (the SC group, namely the group using stick crayons). The second group received the same task instructions as the SC group, but were given a different type of crayon to use. They were provided with block crayons, in the same colour range as the SC group, typically employed by children attending Steiner (Steiner, 1974, 1985) 
schools. The crayons are rectangular blocks providing a range of edges and sides which can be used to produce different types and sizes of marks. The crayons are used across the Steiner school system in order to afford children the opportunity to make a greater range of strokes and shaded marks than more conventional stick crayons. In contrast to mainstream schooling across the UK, Steiner education is based upon its own theory of child development (Nicholson, 2000, Woods \& Woods, 2002) and exploration with different media of artistic expression is a core aspect of artistic development (Nicholson, 2000; Woods, Ashley \& Woods, 2005). Because of the different size and the different drawing techniques which the blocks afford to the child, it was anticipated that the strategies children in this group would use would be of a greater range than those produced by children employing stick crayons.

\section{Drawing strategies}

The study was therefore designed to investigate whether a change in drawing materials influences a different pattern of strategy use in children's drawings of positive and negative men. Two lines of prediction were open. If task materials do influence children's drawings, then group differences would be expected. However, if children's drawings are less sensitive to manipulations in task material, and are instead influenced more by cognitive representations or by representational and/or strategic intentions, no differences between the experimental groups would be expected in the strategies used to depict positive and negative men. In addition, in line with previous findings by Burkitt et al. (2003a, 2004), the aim was to explore whether positive men would be drawn larger than baseline and negative men, irrespective of materials, or whether drawing materials would interact with children's age and gender in their impact on drawing size.

\section{Colour use}


Children tend to choose more preferred colours for positively characterised men, less preferred colours for baseline men, and less preferred colours still for negatively characterised men (Burkitt et al., 2003b, 2004). As the block and stick crayons here were provided in the same colour range, it was anticipated that the pattern of colour use would continue and not be sensitive to the type of medium provided. Children's use of particular colours for neutral, positive and negative men was also explored, as were potential interactions between age and gender. It was anticipated that children's colour preferences would follow the same general trend as in former studies, namely a preference for primary colours, followed by secondary colours, with the achromatic range including brown being the least preferred.

\section{Method}

Participants

132 children from two mainstream schools in Surrey and Sussex UK participated in this study. They were selected from age appropriate schools and year groups to form three age groups and alternately allocated into two groups from class lists. 66 children (36 boys and 30 girls) formed the group using stick crayons (SC group) and a further 66 children (33 boys and 33 girls) were given block crayons and formed the BC group. The age and age range of the children are shown in Table 1.

$$
\text { **INSERT TABLE } 1 \text { ABOUT HERE** }
$$

\section{Procedure}

All children were seen individually in a quiet area of their school and completed two sessions which were administered in counterbalanced order. The only variation between the procedures was the provision of block crayons for one group (BC group) and stick crayons for the other group (SC group). To reduce the possible influence of 
practice effects, children in the $\mathrm{BC}$ group were given an opportunity to familiarise themselves with the media prior to testing. Children were given a separate piece of A4 rectangular plain white paper to use for each drawing which was placed in front of them in a portrait orientation. None of the children reoriented the paper to a landscape orientation when completing the drawings. There was no set time for drawing completion and children were free to embellish the drawings if they chose to.

Session 1. In Session 1, all children completed a colour preference task. The children were shown the ten colour cards in a random order. As each colour was presented, they were asked to rate how the colour made them feel, using the 5-point Likert scale. Responses were scored between 1 and 5, with $1=$ "very unhappy" and $5=$ "very happy". The instructions were as follows: "I would like to find out how you feel about this colour. What I'd like you to do is point to the face to show how you feel about the colour. Here are the faces that you are going to be looking at (pointing to each face). The first one is a very unhappy face; the next one is quite an unhappy face; the middle one is neither happy nor unhappy. The fourth face is quite a happy face and the last one is a very happy face. When you answer my question, I'd like you to point to the face that describes how you feel about the colour. OK?" The instructions were repeated in full if the child indicated that they had not understood, but few children required additional prompts.

Session 2. In session 2, children drew three pictures of a man. Children were asked to draw male rather than female human figures partly for consistency with most previous research in this field and partly to eliminate the possibility that children would make size changes in response to the gender of the drawn figure, as previous research has found that females are sometimes drawn larger than males in order to facilitate the inclusion of specific features such as long hair and dresses (Cox, 1995; Davis \& 
Arazos, 1989) rather than changing size in relation to the affect they held towards the drawn figure. All children drew a baseline figure first to which no affective characterisation was associated. They then drew a positive and a negative man in counterbalanced order.

Children in both groups were asked to draw the baseline, neutrally characterised, figure using the following instructions: "I'd like you to draw a man. Use the crayon to draw him, and just one of these colours to colour him in. Draw the whole man as well as you can and colour him in as well as you can”.

Both groups were given the following instructions for each characterised figure. Positive figure: "Now, think of a man who is a very very happy man, and who is very pleasant and friendly to everyone. Draw the man, remembering what a happy person he is. Use just one of these colours to colour him in. Draw the whole man as well as you can and colour him in as well as you can". Negative figure: "Now, think of a man who is a very very sad man, and who is very mean and unfriendly to everyone. Draw the man, remembering what a sad man he is. Use just one of these colours to colour him in. Draw the whole man as well as you can and colour him in as well as you can". Immediately after drawing each individual figure, each child was asked to rate how he or she felt about the characterised figure (not the actual drawing) by pointing to the appropriate face on a five point Likert scale. The rating system was the same as that used to measure how children felt about the colours they had used in their drawings. Responses were scored between 1 and 5, with $1=$ "very unhappy" and $5=$ "very happy". The drawing was then removed, and the child was given a fresh sheet of A4 paper for the production of the next drawing. The same range of crayons were left in place throughout the session. 
Drawing ability. The six class teachers were asked to rate each child's drawing ability relative to a typical year group member's ability using the following question: "Thinking of a typical Year \{Year group of child\}, please rate \{child's name\} drawing ability on the following scale: poor (1), below average (2), average (3), above average (4), good (5). 'This measure was taken to check that, at any given age, the participating children were of comparable level of drawing ability between the two experimental groups.

Measurements. All children successfully completed the range of tasks. The height of each drawing was measured as the vertical distance from the top to the lowest extremity of the outlines. Width was measured as the horizontal distance between the farthest left and right extremities of the outline of the figure.

Data analysis. ANOVAs followed up with post hoc t-tests were conducted to ascertain whether there were any differences in drawing strategies as a function of age, gender, type of materials and drawing type, and whether there were any differences in how the children felt about the figures they had drawn and about the colours they had used for each drawing type as a function of the same four factors. Correspondence analysis was used to see if any associations between specific colours, drawing type and type of materials were evident.

\section{Results}

To assess any differences in drawing ability between the children in the different experimental groups, drawing ability was analysed using a 3 (age group) x 2 (gender) x 2 (group) ANOVA. No significant main or interaction effects were found, indicating that there were no significant differences between the experimental groups in terms of drawing ability (overall $\mathrm{M}=3.8, \mathrm{SD}=0.27$ ). All children were indeed able to 
draw a male human figure. This factor was thus excluded from further analysis. The data were also examined for possible order effects, and none were found. Thus, order was excluded from the following analyses.

Drawing size

A series of ANOVAs was conducted to assess whether drawing size (height and width) varied as a function of age, gender, material type or drawing type.

Height. A 3 (age group) x 2 (gender) x 2 (group) x 3 (drawing type) four-way mixed ANOVA was conducted, with drawing type entered as the repeated measure, and independent measures on the other three factors. A main effect was found for drawing type $(F(2,240)=18.41, p<0.01)$. Observed power was high for this effect $(P=1.00$, partial eta $\left.{ }^{2}=0.13\right)$. Post hoc paired t-tests $(p<0.05)$ on the means in Table 2 showed that positive drawings were taller than both the baseline and negative drawings, and negative drawings were also taller than baseline drawings. A small main effect with fairly high power $\left(P=0.06\right.$, partial eta $\left.{ }^{2}=0.04\right)$ was found for gender $(F(1,120)=4.53$, $p<0.05)$, and the means in Table 2 show that boys drew taller overall than girls. An equally small interaction effect with relatively high observed power $(P=0.77$, partial eta $\left.^{2}=0.04\right)$ between drawing type and gender was found $(F(2,240)=4.53, p<0.05)$. Post hoc independent and paired t-tests $(p<0.05)$ showed that the boys drew taller positive and negative drawings than the girls, but there were no differences between boys and girls for the height of baseline drawings. Both the boys and the girls drew positive men taller than the baseline and negative drawings, yet only the boys drew significantly taller negative than baseline men.

\section{**INSERT TABLE 2 ABOUT HERE**}

A main effect for group was also found $(F(1,120)=27.29, p<0.05)$. The observed power was high for this medium effect size $\left(P=1.00\right.$, partial eta $\left.^{2}=0.25\right)$. 
Inspection of the means revealed that the $\mathrm{BC}$ group produced taller drawings overall than the SC group (BC: $M=18.00, S D=5.91$ vs. $\mathrm{SC}: M=13.08, S D=4.88$ ). No further main or interaction effects for drawing height were found.

Width. A 3 (age group) x 2 (gender) x 2 (group) x 3 (drawing type) four-way mixed ANOVA was conducted, with drawing type entered as the repeated measure, and independent measures on the other three factors. A small main effect was found with high observed power $\left(P=0.95\right.$, partial eta $\left.{ }^{2}=0.06\right)$ for drawing type $(F(2,240)=7.93$, $p<0.01)$, and post hoc paired t-tests $(p<0.05)$ on the means in Table 3 revealed that the positive drawings were wider than both the baseline and negative drawings. A small interaction effect with high observed power $\left(P=0.92\right.$, partial eta $\left.{ }^{2}=0.07\right)$ was found between drawing type and age group $(F(4,240)=4.20, p<0.05)$. Post hoc paired t-tests $(p<0.05)$ on the means in Table 3 showed that for the youngest group, both baseline and positive drawings were wider than negative drawings, no significant differences emerged for the middle age group's drawing size, and for the oldest age group, both positive and negative drawings were wider than the baseline drawings.

\section{**INSERT TABLE 3 ABOUT HERE**}

A second main effect was found for group $(F(1,120)=48.49, p<0.01)$. The observed power was high for this medium sized effect $\left(P=1.00\right.$, partial eta $\left.^{2}=0.30\right)$. The means in Table 4 show that children in the BC group drew wider drawings than the children in the SC group. An interaction effect was found between drawing type and group $(F(2,240)=3.47, p<0.05)$. Post hoc paired t-tests $(p<0.05)$ revealed that the SC group drew significantly wider positive than negative and baseline drawings, while no significant differences were found between the three BC drawing widths. 
A content analysis was performed by two adult judges on the children's drawings for the strategies which were used for showing differences between the negatively and positively characterised figures and the neutrally characterised figures. The categories were derived directly from the data. The emergent categories (which obtained a 95\% inter-judge agreement overall for their presence or absence) were as follows (percentages in parentheses represent the level of inter-judge agreement for the presence or absence of each category):

- Details (96\%): This category included using specific core features of the figure to denote positivity or negativity, for example the inclusion of facial features such as a smile or frown, and clothing features such as love hearts and flowers on jumpers for positive drawings or chain-mail for negative drawings.

- Actions (94\%): This category included drawings where actions were depicted, for example, figures laughing or crying.

- Use of Line (84\%): Drawings in this category included those where elements had been drawn and/or coloured neatly or messily. For example, some children reported that they were deliberately scribbling over the outlines of the negative drawings when colouring the figures.

- Use of colour (100\%): Drawings where the colour was changed in the children's drawings of the characterized figures. Children frequently stated that they had used "pretty", "happy" or their "favourite" colours for the positive drawings, and "ugly", "dead" or "hated" colours for the negative drawings.

- Size variations (94\%): This category included instances where children were judged to be using size as a device, whether it be by producing small or large drawings. 
- Directional size change (96\%): This category included drawings where positively characterized figures were larger than negatively characterized figures. For example, children frequently said that nice men are "more cuddly" than nasty men who are "skinny".

- Mutations (90\%): Examples of drawings in this category included children altering or exaggerating certain features of a figure. For example, some children drew peculiarly shaped negative men to emphasise their negativity.

- Words (100\%): Children sometimes included words (either within or without speech bubbles) or names for figures to show that the figure was positive or negative. For example, one child showed the positive figure saying the word "cool". Wording on clothing was also included in this category.

- Characterisations (94\%): Drawings were included in this category if the figures were drawn as specified characters. Children sometimes drew specific cartoon figures, for example, Superman. Specified figures known to the children were also included in this category, for example, a favoured parent or football player.

- Line quality (91\%): Drawings were scored in this strategy, for example, when a heavy or a feint line was used in drawings of the positive and negative figures.

This range of strategies replicates that found in a previous study (Burkitt \& Barrett, 2010). For each category of strategy separately, each characterised drawing was then scored as 1 or 0 in order to identify the strategies which the children had actually used in their drawings of positive and negative figures in order to show that the figure was different from the neutral baseline figure.

The scores for each category were separately analysed using 3 (age group) x 2 (gender) $\times 2$ (group) x 2 (drawing type) four-way mixed ANOVAs to inspect for 
potential drawing type, group, age and gender differences in the use of each strategy. ${ }^{1}$ No significant main or interaction effects were found for the strategies of line use or word use. However, there were significant effects on all of the following strategies.

Details. ANOVA revealed a small main effect with fairly high observed power $\left(P=0.77\right.$, partial eta $\left.{ }^{2}=0.08\right)$ for age group $(F(2,120)=4.55, p<0.05)$. Post hoc Tukey $(p<0.05)$ analysis indicated that more use of detail was observed in the drawings of the oldest age group $(M=1.00, S D=0.00)$ compared with the youngest group $(M=0.86$, $S D=0.33)$. No additional main or interaction effects were found.

Actions. ANOVA revealed a main effect for age group $(F(2,120)=6.79$, $p<0.05)$. Observed power was high for this small effect $\left(P=0.91\right.$, partial eta $\left.^{2}=0.10\right)$. Post hoc Tukey $(p<0.05)$ analysis indicated that more use was observed for the oldest compared $(M=0.30, S D=0.36)$ with the youngest age group $(M=0.09, S D=0.19)$. No additional main or interaction effects were found.

Use of colour. ANOVA indicated a main effect for age group $(F(2,120)=$ $6.49, p<0.05)$, with high observed power and small effect size $\left(P=0.91\right.$, partial eta $^{2}=$ 0.11). Post hoc Tukey testing $(p<0.05)$ on the means revealed that more use was observed overall for the middle age group $(M=0.97, S D=0.13)$ compared with the youngest age group $(M=0.77, S D=0.37)$. No further main or interaction effects were found.

Size variations. A small main effect with fairy high observed power $(P=0.79$, partial eta $\left.^{2}=0.06\right)$ was found for group $(F(1,120)=7.23, p<0.05)$. Inspection of the means showed that more use was observed in the drawings from the $\mathrm{BC}$ group $(M=$

\footnotetext{
${ }^{1}$ Although ANOVA is not normally used to analyse dichotomous data, this is a well-established statistical procedure for analysing such data which produces accurate results when there are at least 20 degrees of freedom for error (Lunney, 1970) and the dichotomous data are recorded in binary format (Gabrielsson \& Seeger, 1971).
} 
$1.00, S D=0.00)$ than the $S C$ group $(M=0.92, S D=0.22)$. There were no additional main or interaction effects for size variations.

Directional size use. ANOVA revealed a main effect for drawing type ( $F$ $(1,120)=17.81, p<0.01)$. The observed power was high and the effect size was small $\left(P=0.99\right.$, partial eta $\left.^{2}=0.13\right)$. The means are shown in Table 5 , and show that more use was observed in children's positive drawings as scaled up from baseline drawing size. A small interaction effect between drawing type and group was found with medium observed power $\left(P=0.56\right.$, partial eta $\left.{ }^{2}=0.05\right)(F(1,120)=4.57, p<0.05)$. Post hoc paired and independent t-tests $(p<0.05)$ on the means in Table 5 showed that more use was only observed in the positive compared with the negative drawings for the SC group, and that for the negative drawings greater use was observed in the $\mathrm{BC}$ group.

**INSERT TABLES 5 AND 6 ABOUT HERE**

An interaction effect between drawing type and gender was also found $(F(1,120)=$ 6.68, $p<0.01)$. The observed power was fairly high for this small effect $(P=0.73$, partial eta ${ }^{2}=0.05$ ). Table 6 displays the means, and post hoc t-tests revealed that more use was observed in the positive compared to negative drawings for the boys, but not significantly for the girls. It was also found that for the positive drawings more use was observed for boys, whilst for the negative drawings, more use was observed for the girls. No further main or interaction effects were found.

Mutation. ANOVA revealed a small main effect with fairly high power $(P=$ 0.66 , partial eta $\left.{ }^{2}=0.01\right)$ for group $(F(1,120)=4.92, p<0.05)$ with the means showing that mutations were used more by the BC group $(M=0.63, S D=0.39)$ than the SC group $(M=0.48, S D=0.40)$. A main effect was also found for age group $(F(2,120)$ $=4.34, \mid p<0.05)$. Observed power was high for this large effect $\left(P=0.74\right.$, partial eta $^{2}=$ 0.70). Post hoc Tukey testing $(p<0.05)$ on the means revealing that more mutation use 
was observed for the middle age group $(M=0.67, S D=0.39)$ compared with the youngest age group $(M=0.43, S D=0.40)$. No additional main or interaction effects were found.

Characterisations. ANOVA revealed a main effect for drawing type $(F(1,120)$ $=6.67, p<0.05)$. The effect was small and the observed power was fairly high $(P=$ 0.73 , partial eta $\left.{ }^{2}=0.05\right)$. More use was observed in the positive $(M=0.25, S D=0.43)$ compared with the negative $(M=0.16, S D=0.37)$ drawings. A main effect was also found for age group $(F(2,120)=5.92, p<0.05)$. Post hoc Tukey testing $(p<0.05)$ indicated that more use was observed for the oldest group $(M=0.35, S D=0.44)$ compared with both the middle $(M=0.16, S D=0.28)$ and youngest $(M=0.10, S D=$ $0.25)$ age groups. No further main or interaction effects were found.

Line quality. A small main effect with high observed power $(P=1.00$, partial eta $\left.^{2}=0.16\right)$ was found for drawing type $(F(1,120)=22.52, p<0.05)$. The means in Table 7 indicate that more use was observed in children's negative drawings. An interaction effect was found between drawing type and group $(F(1,120)=5.86$, $p<0.05)$. The observed power was fairly high and the effect size was small $(P=0.66$, partial eta $\left.{ }^{2}=0.05\right)$. Post hoc paired t-tests $(p<0.05)$ revealed that more use was observed in the negative compared with the positive drawings for the SC group. Post hoc independent t-tests $(p<0.05)$ showed that for the positive drawings, significantly more use was observed for the $\mathrm{BC}$ group, whereas for the negative drawings, more use was observed for the SC group. No additional main or interaction effects were found.

**INSERT TABLE 7 ABOUT HERE**

Affect towards neutrally, positively and negatively characterised figures

The ratings from the Likert scale gathered in Session 2 towards the three drawn figures were analysed to investigate whether children assigned different ratings to the 
differently characterised figures. A 3 (age group) x 2 (gender) x 2 (group) x 3 (drawing type) four-way mixed ANOVA was conducted, with drawing type entered as repeated measure, and independent measures on the other three factors.

A large main effect with high observed power $\left(P=1.00\right.$, partial eta $\left.^{2}=0.75\right)$ was found for drawing type $(F(2,240)=361.01, p<0.01)$, and post hoc paired t-tests revealed that more positive ratings were given to the positive figures $(M=4.37, S D=$ $0.97)$ than to the negative $(M=1.34, S D=0.66)$ and baseline $(M=2.94, S D=0.98)$ figures, and that the baseline figures were also rated significantly more positively than the negative figures. No additional main or interaction effects were found.

Affect towards the colours chosen for the three drawing types

The ratings from the Likert scale gathered in Session 1 towards the colours children chose to complete the three drawings in session 2 were analysed to investigate whether children used different colours for the three drawing types, and whether they rated the colours which they chose differently for the three drawings. A 3 (age group) $x$ 2 (gender) x 2 (group) x 3 (drawing type) four-way mixed ANOVA was conducted, with drawing type entered as the repeated measure, and independent measures on the other three factors.

A main effect was found for drawing type $(F(2,240)=82.57, p<0.01)$. The size of the effect was relatively large and observed power was high $(P=1.00$, partial eta $\left.^{2}=0.41\right)$. Post hoc paired t-tests $(p<0.05)$ on the scores showed that the colours chosen for the positive drawing were given more positive ratings $(M=4.52, S D=0.86)$ than both the baseline $(M=3.90, S D=1.09)$ and negative drawing $(M=2.73, S D=$ 1.49) colour choices, and that the baseline colour was given more positive ratings than the colour chosen for the negative drawings. No additional main or interaction effects were found. 
Use of specific colours

Children's use of particular colours for each drawing type was analysed using correspondence analysis. Correspondence analysis (Hammond, 1988, 1993) uses geometric principles to provide a pictorial representation of the relationship between categories of response and groups of individuals. It performs a multidimensional analysis of categorical data, and provides a plot in which the geometric distance between the groups and the types of response reflects the relative degree of association between the groups and the response types. This graphical representation therefore shows those colour choices which are most closely associated with each group (be it age, gender, condition or drawing type) and which therefore best discriminate the behaviour of the children in each subgroup. Separate analyses were run for each drawing type, and broken down separately by group, age and gender. No effects emerged for group differences between baseline and positive drawings; however, there were group differences between the colours selected for the negative men (one dimensional solution: $\left.\chi^{2}(9)=28.85, p<0.05\right)$. Green and orange were more closely associated with the BC group (used by $29 \%$ and $37 \%$ of the children in this group, respectively) than with the SC group (used by $13 \%$ and 10\%, respectively) in their drawings of negative men. The two most frequently used colours for the baseline drawings overall were red (18\%) and blue (31\%), for the positive drawings were red (31\%) and yellow (20\%), and for the negative drawings were purple (24\%) and black $(35 \%)$.

\section{Discussion}

Use of differential size as a drawing strategy 
The present study has provided some evidence to suggest that previous findings using positive and negative characterisations (e.g. Burkitt et al., 2003a, 2004; Burkitt \& Newell, 2005; Cleeve \& Bradbury, 1992) are generalisable to drawing tasks where children are asked to use different drawing materials. Regardless of type of drawing material, in line with previous findings (Aronsson \& Andersson, 1996; Cleeve \& Bradbury, 1992; Craddick, 1961; Fox \& Thomas, 1990; Thomas et al., 1989), positively characterised men were drawn taller than uncharacterised and negative men, and negative men were not reduced in size from uncharacterised men. In this sample of 4 to 7 year old children, the youngest group drew wider positive than negative men, and the oldest group drew wider positive than negative and baseline men. These effects seem robust and independent of the drawing materials provided.

Also in line with previous findings regardless of material used (Craddick, 1963; Jolley, 1995; Thomas, Chaigne \& Fox, 1989), size changes following negative topic characterisation seem less consistent under experimental conditions (e.g. Burkitt \& Barnett, 2006; Jolley, 1995). For drawing width, whilst the older children drew both uncharacterised and happy men wider than negative men, the youngest group also reduced the width of the negative men from baseline size. It seems as though changes in width are more sensitive to the experimental manipulation of emotional character, but not in a consistent way, and not due to any influence of drawing materials.

Boys produced bigger drawings than girls. In the current study, however, this occurred with drawing height, but not with drawing width as has occurred in previous research (e.g. Burkitt et al., 2003a, 2004). Thus, although a consistent pattern is emerging of boys producing bigger drawings independent of the emotional character of the figure than girls, this may not always occur in all dimensions. It could be that motor 
control and planning varies between the genders depending on the sequencing of how the drawing is produced.

The present findings lend support to proponents of the conceptual theory that children's drawings of particular scenes or objects relate to their concepts of the object (Goodenough, 1926; Harris, 1950; Luquet, 1913, 1927; Piaget \& Inhelder, 1956, 1969); thus some aspects of children's drawings remain consistent across drawing tasks. It could be argued that within children's mental representations of positive men, information about figure size is encoded independently of motor and planning factors. This information may also include the coding that positive figures are to be drawn relatively larger than negative figures. Alternatively, as suggested by Thomas et al. (1989), it could be the case that children are responding on the basis of a mechanism which leads them to exaggerate the size of positive topics to increase the appeal of the drawn figure.

\section{Differences associated with type of drawing materials}

The manipulation of drawing materials did, however, affect some size differences between children's drawings of positive and negative men. The positive figures were drawn wider than both the baseline and negative figures when stick crayons were used, yet this was not the case when the block crayons were used. This finding may reflect the differential task demands imposed by the use of different drawing materials, offering support to the position that drawings are influenced by the exact task demands of the situation, including the type of materials employed. It could be argued that negotiating the use of the block crayons overrode the children's considerations of employing the strategy of size (width) to differentiate the emotional character of the figure. Again it seems that drawing width occupies a different place when children are negotiating drawing from drawing height. 
The children using block crayons produced larger and taller drawings than the children using the Crayola stick crayons. This finding probably reflects the nature of the drawing materials, as it is possible to produce broader marks with block crayons than with stick crayons by using the extra flat surfaces which the block crayons provide. These findings are in line with the proposition (Gardner, 1978; Golomb, 1973) that drawing construction is at least partly determined by the differential demands placed upon children as a consequence of the cues resulting from differing drawing materials.

The above results indicate that the type of media employed interacts with some aspects of children's drawings, but not others (Brittain, 1986; Brittain \& Chein, 1980). Although there were influences of age and gender, as no major age-related trends were found to interact with the type of drawing materials, or with the type of drawing being constructed, there is little support for the idea that drawing materials interact with children's developmental level under these conditions.

\section{The range of drawing strategies, and links with drawing materials}

The same range of categories of drawing strategies was found for both groups of children, and replicated the range of strategies found in past research (Burkitt \& Barrett, 2010; Burkitt et al., 2004). There were differences between the age groups in the use of certain strategies, but this was unrelated to the types of drawing materials which the children were given. Older children used more details, actions, colour changes, mutations and characterisations than the younger children. Such differential use is in agreement with the literature showing that children's use of representational techniques improves with age (e.g. Cox, 1992; Hammer, 1997; Ives, 1984; Sayil, 1996, 1998; Winston et al., 1995) and is concurrent with age-related trends in the use of these strategies as seen in previous research (Burkitt et al., 2004). 
However, there were some differences in the ways that the children using stick crayons and the children using block crayons employed particular strategies. Changes in size were observed more in the drawings from children using the block crayons. This is in line with the quantitative measurement data, and probably reflects the previously described differences between the drawing materials. More use of directional size change was observed for the positive drawings compared with the negative drawings for the children using stick crayons, whereas children using the block crayons employed this strategy more when drawing negative as opposed to positive drawings. More use of the strategy of mutation occurred with the children using block crayons. A possible explanation for this finding may reflect the greater affordance of exaggerated lines from the shape of the block crayons.

For the strategy of line quality, for the positive figures, the children using the stick crayons exhibited greater use than the children using block crayons, whereas the opposite held for the negative drawings, with the children using the block crayons employing this strategy more than the children using the conventional stick crayons. It is perhaps not surprising that the groups differed in their use of the technique of line quality, as although the stick crayons could be used to produce the same types of lines as the block crayons, this potential was perhaps more obvious to the children using block crayons given the shape of the crayons. The differences in use of size may be attributable to the nature of the crayons, in that the block crayons are larger than the stick crayons. However, this does not explain why the techniques of mutations and line quality were employed differently by the children. These differences might therefore reflect differences in the task demands and interpretation of those task demands as a function of the type of drawing materials used (e.g. Arrowsmith et al., 1994; Barrett et al., 1985; Gardner, 1978; Golomb, 1973). 


\section{Colour use}

The overall anticipated trend for more positively rated colours to be used for positive figures than for both baseline and negative figures was unaffected by the use of materials. No particular colours were associated with each drawing type when the groups' responses were analysed separately. This finding may indicate that children's use of the drawing materials overrode decisions to employ different colours for the different drawing types. However, when the drawings of negative men were analysed separately between the groups, children's colour response were discriminated. As the block and stick crayons were provided in the same colour range, this effect implies that this variation in task demands exerts a different effect on children' colour choices for drawing negative men than for drawings of neutral and positive men. Use of particular colours for baseline and positive figures did not differ significantly between the groups. The order of colour preference was unsurprisingly broadly unaffected by drawing material, and children's order of colour preference followed the same general trend as with the control group (SC) and previous results (Burkitt et al., 2003b, 2004; Burkitt \& Newell, 2005; Gelineau, 1981; Nelson et al., 1971), with primary colours rated most positively, followed by secondary colours and the achromatic range respectively. It appears that colour use is less affected by variations of drawing material than drawing size.

\section{Conclusions}

This experiment has provided evidence to suggest that some aspects of children's drawings of positive and negative men are independent of changes in drawing materials, but that other aspects of their drawings are affected by the drawing materials which are used. The absence of age differences in the use of size and colour 
suggests that if children are responding to these aspects using pictorial conventions, these are acquired at an early age.

Children followed the same pattern of colour use in relation to colour preference, and used the same range of additional strategies as has been found in previous experiments, and these were independent of the types of crayons they were using. This lends support for the generalisability of previous findings, and the view that some aspects of children's drawings of certain objects remain similar across tasks where drawing materials are varied.

However, the drawing materials were found to sometimes interact with drawing type in influencing aspects of the characterised men which were drawn. For example, the drawing materials influenced the width of the characterised men in interaction with the type of emotional character which children were attempting to represent. It could be that children's preoccupation with the drawing materials detracted from their conception of how to represent emotional character. In addition, the use of mutations and line quality for representing positive and negative figures varied depending on the drawing materials which the children were using.

These findings suggest that not only do practitioners need to take into account children's attitude towards the figure which they are drawing, but also the type of materials which are being used and the particular aspect of the drawing under consideration, when interpreting children's drawings of positive and negative men. For example, it may be erroneous to conclude that a child feels positively about a large figure they have drawn if the size of the drawing is simply a result of the type of implement they have used. It is important that the exact cues present in a drawing situation are known before conclusions about the emotional content of a child's drawings are made. Freeman (1995) argued that the precise cues present in individual 
drawing tasks precisely determine the form of the resultant drawing, and it seems that material types, even with such a slight manipulation of crayon type, can influence differences in relation to certain drawing strategies such as drawing size which are commonly believed to indicate positive and negative affect towards the topics children draw. The implications of the present research are that practitioners need to understand precisely how the use of different media does and does not influence expressive aspects of children's drawings, cautioning against the interpretation of the significance children's drawings without taking into account the specific type of medium used within particular drawing activities.

\section{Limitations and future directions}

One possible limitation of the present study was that a between-subjects rather than a within-subjects design was used. However this approach was taken because it was anticipated that doubling the number of drawings could result in participant fatigue which would lead to a deterioration in performance on later drawings, and because it was anticipated that order effects could be large if a within-subjects design were to be used (it is well established that order effects commonly occur in children's drawings: Cox, 1992; Freeman, 1995).

Secondly, even though the children were given a period to familiarise themselves with the block crayons, it could be argued that practice effects contributed to the performance of the children using the more familiar stick crayons. The relative familiarity of children with the stick crayons may have enhanced the production of smaller, more controlled drawings. It was however observed that children did not appear to have difficulties with the block crayons following their period of familiarisation to the types of marks which the crayons afforded, and no children 
complained of any difficulties with using the crayons during the test sessions. It could also be argued that the block crayons are not intrinsically difficult to use, as very young children manage to exact sufficient motor control when using the chubby crayons typically seen in infant and primary schools. The present experiment does however suggest that further empirical work is necessary to determine the role of drawing materials on drawing performance.

This line of research could be extended in various ways. Children's portrayal of emotionally salient stimuli could be further explored by directly comparing the use of different types of materials, for example paints, and other representational media such as clay, fuzzy felt and wooden puzzles. This would help to inform the debate about the circumstances under which children's representational abilities are influenced by the nature of the materials provided, and would offer further insights about how children's representations of different kinds of emotionally salient figures are differentially influenced as a function of the media employed. A much wider range of emotional labels could also be used in future research, to establish the extent to which different emotions interact with the materials children use to depict affectively characterised topics, and to examine whether it is only the positive/negative dimension of emotions which is important here or whether there are more specific effects associated with different emotions. 


\section{References}

Alschuler, R., \& Hattwick, L. A. (1943). Easel painting as an index of personality in pre-school children. Journal of Orthopsychiatry, 13, 616-625.

Alschuler, R., \& Hattwick, L. A. (1947). Painting and personality. Volumes I and II. Chicago: The University of Chicago Press.

Arnheim, R. (1956). Art and visual perception: A psychology of the creative eye. London: Faber \& Faber.

Aronsson, K. \& Andersson, S. (1996). Social scaling in children's drawings of classroom life: A cultural comparative analysis of children's drawings in Africa and Sweden. British Journal of Developmental Psychology, 14, 301-314.

Arrowsmith, C. J., Cox, M. V. \& Eames, E. (1994). Eliciting partial occlusions in the drawings of 4- and 5- year olds. British Journal of Developmental Psychology, 12, $577-$ 584.

Barrett, M. D. \& Bridson, A. (1983). The effect of instruction upon children's drawings. British Journal of Developmental Psychology, 1, 175-178.

Barrett, M. D. \& Eames, K. (1996). Sequential developments in children's human figure drawings. British Journal of Developmental Psychology, 14, 219-236.

Barrett, M. D. \& Light, P. H. (1976). Symbolism and intellectual realism in children's drawings. British Journal of Educational Psychology, 46, 198-202.

Barrett, M. D., Sutherland, A. \& Lee, F. (1987). Visual realism in children's drawings: The effects of instructions. Paper presented at the British Psychological Society Developmental Section Annual Conference, York, September. 
Brittain, W. L. (1986). The effect of materials on young children's drawings. Visual Arts Research, 12, 10-15.

Brittain, W. L. \& Chien, Y.C. (1980). Effect of materials on preschool children's ability to represent a man. Perceptual and Motor Skills, 51, 995-1000.

Burkitt, E. and Barnett, N. (2006). The effects of brief and elaborate mood induction procedures on the size of young children's drawings. Educational Psychology, 26, 93108.

Burkitt, E. \& Barrett, M. (2010). Children's graphic flexibility: A response to representational redescription, Journal of Creative Behavior,44, 169-190.

Burkitt, E., Barrett, M. \& Davis, A. (2003a). The effect of affective characterisations on the size of children's drawings. British Journal of Developmental Psychology, 21, 565584.

Burkitt, E., Barrett, M. \& Davis, A. (2003b). The effect of affective characterisations on the use of colour within children's drawings. Journal of Child Psychology and Psychiatry, 44, 3, 445-455.

Burkitt, E., Barrett, M. \& Davis, A. (2004). The effect of affective characterizations on the use of size and colour in drawings produced by children in the absence of a model. Educational Psychology, 24, 3, 315-343.

Burkitt, E. \& Newell, T. (2005). The impact of topic specificity on children's depiction of emotional traits. International Journal of Art Therapy, 10, 1, 15-22.

Callaghan, T. C. (1999). Early understanding and production of graphic symbols. Child Development, 70, 1314-1324. 
Chen, M. J. \& Holman, J. (1989). Emergence of drawing devices for total and partial occlusions: A longitudinal study. Perception, 18, 445-455.

Cleeve, H. \& Bradbury, R. J. (1992). Children's productions and judgements of drawings of people of different importance. Poster presented at the 5th European Conference of Developmental Psychology, Seville, September.

Cox, M. V. (1981). One thing behind another: Problems of representation in children's drawings. Educational Psychology, 1, 275-287.

Cox, M. V. (1985). One object behind another: Young children's use of array-specific or view-specific representation. In N. H. Freeman \& M. V. Cox (Eds.), Visual order: The nature and development of pictorial representation, pp. 188-200. Cambridge: Cambridge University Press.

Cox, M. V. \& Parkin, C. (1986). Young children's human figure drawing: Crosssectional and longitudinal studies. Educational Psychology, 6, 353-368.

Craddick, R. (1961). Size of Santa Claus drawings as a function of time before and after Christmas. Journal of Psychological Studies, 12, 121-125.

Craddick, R. A. (1963). Size of Hallowe'en witch drawings prior to, on and after Hallowe'en. Perceptual and Motor Skills, 16, 235-238.

Davis, A. M. (1983). Contextual sensitivity in young children's drawings. Journal of Experimental Child Psychology, 35, 478-486.

Davis, A. M. (1985a). Conflict between canonicality and array-specificity in young children's drawings. British Journal of Developmental Psychology, 3, 363-372. 
Davis, A. M. (1985b). The canonical bias: young children's drawings of familiar objects. In N. H. Freeman \& M. V. Cox (Eds.), Visual order: The nature and development of pictorial representation, pp. 202-213. Cambridge: Cambridge University Press.

Davis, A. M. \& Arazos, A. (1989). Young children's representation of gender in drawings. Paper presented at the Annual Conference of the British Psychological Society Developmental Section, University of Surrey.

Davis, A. M. \& Bentley, M. (1984). Young children's interpretation of the task demands in a simple experimental situation: An example from drawing. Educational Psychology, 4, 249-254.

Fenson, L. (1985). The transition from construction to sketching in children's drawings. In N. H. Freeman \& M. V. Cox (Eds.), Visual order: The nature and development of pictorial representation, pp. 374-384. Cambridge: Cambridge University Press.

Fox, T. \& Thomas, G. V. (1990). Children's drawings of an anxiety-eliciting topic: Effect on size of the drawing. British Journal of Clinical Psychology, 29, 71-81.

Freeman, N. H. (1995). The emergence of a framework theory of pictorial reasoning. In C. Lange-Kuttner (Ed.), Drawing and looking: Theoretical approaches to pictorial representation in children, pp. 135-146. Hemel Hempstead: Harvester Wheatsheaf.

Gabrielsson, A. \& Seeger, P. (1971). Tests of significance in two-way designs (mixed model) with dichotomous data. British Journal of Statistical Psychology, 24, 111-116.

Gardner, H. (1978). Developmental psychology: An introduction. Boston: Little Brown. 
Gelineau, E. P. (1981). A psychometric approach to the measurement of color preference. Perceptual and Motor Skills, 53, 163-174.

Goodenough, F. L. (1926). The measurement of intelligence by drawings. New York: World Books.

Goodnow, J. (1977). Children's drawings. London: Open Books.

Hammer, E. F. (1953). Frustration-aggression hypothesis extended to socio-racial areas: Comparison of Negro and white children's H-T-P's. Psychiatric Quarterly, 27, 597-607.

Hammer, E. F. (1997). Advances in projective drawing interpretation. Springfield, Ills.: C. C. Thomas.

Harris, D. B. (1950). Intra-individual vs. inter-individual consistency in children's drawings of a man. American Psychologist, 5, 293.

Ives, S. W. (1984). The development of expressivity in drawing. British Journal of Educational Psychology, 54, 152-159.

Jolley, R. (1995). Children's production and perception of visual metaphors for mood and emotion in line drawings and in art. Unpublished doctoral thesis. University of Birmingham, Birmingham.

Koppitz, E. M. (1968). Psychological evaluation of children's human figure drawings. London: Grune and Stratton. 
Lewis, C., Russell, C. \& Berridge, D. (1993). When is a mug not a mug? Effects of content, naming, and instructions on children's drawings. Journal of Experimental Child Psychology, 56, 291-302.

Leibowitz, M. (1999). Interpreting projective drawings: A self-psychological approach. Philadelphia: Bruner Mazel.

Light, P. H. \& McEwan, F. (1987). Drawings as messages: The effect of a communication game upon production of view-specific drawings. British Journal of Developmental Psychology, 5, 53-60.

Light, P. H. \& Simmons, B. (1983). The effects of a communication task upon the representation of depth relationships in young children's drawings. Journal of Experimental Child Psychology, 35, 81-92.

Lunney, G.H. (1970). Using analysis of variance with a dichotomous dependent variable: an empirical study. Journal of Educational Measurement, 7, 263-269.

Luquet, G. H. (1913). Les dessins d'un enfant. Paris: Alcan.

Luquet, G. H. (1927). Le dessin enfantin. Paris: Alcan.

Machover, K. (1949). Personality projection in the drawings of the human figure. Springfield, IL: C. C. Thomas.

Nelson, T. M., Allan, D. K. \& Nelson, J. (1971). Cultural Differences in the use of color in NorthWest Canada. International Journal of Psychology, 6, 283-292.

Nicholson, D.W. (2000). Layers of experience: Forms of representation in a Waldorf school classroom. Journal of Creative Studies, 32, 575-587. 
Parsons, M. (1995). Young children's representations of friendship in a drawing and non-drawing task. Unpublished undergraduate dissertation, University of Surrey, Guildford, Surrey.

Piaget, J. \& Inhelder, B. (1956). The child's conception of space. London: Routledge \& Kegan Paul.

Piaget, J. \& Inhelder, B. (1969). The psychology of the child. London: Routledge \& Kegan Paul.

Sayil, M. (1996). Preschool children's understanding and drawing of facial expression of emotions. Turkish Journal of Psychology, 11, 61-71.

Sayil, M. (1998). The development of emotional facial drawings in children. Journal of Child and Adolescence Mental Health, 4, 129-133.

Stefanatou, A. \& Bowler, D. (1999). Depiction of pain in the self-drawings of children with sickle cell disease. Child Care, Health and Development, 23, 135-155.

Sitton, R. \& Light, P. (1992). Drawing to differentiate: Flexibility in young children's human figure drawings. British Journal of Developmental Psychology, 10, 25-33.

Steiner, R. (1974). The kingdom of childhood. London: Rudolf Steiner Press.

Steiner, R. (1985). An introduction to Waldorf education. London: Rudolf Steiner Press.

Thomas, G. V., Chaigne, E. \& Fox, T. J. (1989). Children's drawings of topics differing in significance: Effects on size of drawing. British Journal of Developmental Psychology, 7, 321-331. 
Thomas, G. V. \& Jolley, R. P. (1998). Drawing conclusions: A re-examination of empirical and conceptual bases for psychological evaluation of children from their drawings. British Journal of Clinical Psychology, 37, 127-139.

Thomas, G. V. \& Silk, A. M. J. (1990). An introduction to the psychology of children's drawings. Hemel Hempstead: Harvester Wheatsheaf.

Winston, A. S., Kenyon, B., Stewardson, J. \& Lepine, T. (1995). Children's sensitivity to expression of emotion in drawings. Visual Arts Research, 21, 1-14.

Woods, P., Ashley, M. \& Woods, G. (2005). Steiner schools in England. University of the West of England, Centre for Research in Education and Democracy.

Woods, P., \& Woods, G. (2002). Policy on schools diversity: taking an existential turn in the pursuit of valued learning. British Journal of Educational Studies, 50, 254-278. 
Table 1: Age and age range of children using stick (SC) or block crayons (BC)

\begin{tabular}{|c|c|c|}
\hline & SC Group & BC Group \\
\hline $\begin{array}{c}\text { Youngest } \\
\text { Group }\end{array}$ & $\begin{array}{c}(N=22) \\
\text { Mean }=4 y 8 m \\
\text { Range }=4 y 3 m-5 y 2 m\end{array}$ & $\begin{array}{c}(N=22) \\
\text { Mean }=4 \mathrm{y} 7 \mathrm{~m} \\
\text { Range }=4 \mathrm{y} 2 \mathrm{~m}-5 \mathrm{y} 2 \mathrm{~m}\end{array}$ \\
\hline $\begin{array}{l}\text { Middle } \\
\text { Group }\end{array}$ & $\begin{array}{c}\quad(N=22) \\
\text { Mean }=5 y 9 \mathrm{~m} \\
\text { Range }=5 \mathrm{y} 5 \mathrm{~m}-5 \mathrm{y} 11 \mathrm{~m}\end{array}$ & $\begin{array}{c}\quad(N=22) \\
\text { Mean }=5 y 10 \mathrm{~m} \\
\text { Range }=5 \mathrm{y} 7 \mathrm{~m}-6 \mathrm{y} 1 \mathrm{~m}\end{array}$ \\
\hline $\begin{array}{l}\text { Oldest } \\
\text { Group }\end{array}$ & $\begin{array}{c}\quad(N=22) \\
\text { Mean=6y7m } \\
\text { Range }=6 y 3 \mathrm{~m}-7 \mathrm{y} 6 \mathrm{~m}\end{array}$ & $\begin{array}{c}\quad(N=22) \\
\text { Mean }=6 y 7 \mathrm{~m} \\
\text { Range }=6 \mathrm{y} 4 \mathrm{~m}-7 \mathrm{y} 7 \mathrm{~m}\end{array}$ \\
\hline Overall & $\begin{array}{c}\quad(N=66) \\
\text { Mean }=5 \mathrm{y} 10 \mathrm{~m} \\
\text { Range }=4 \mathrm{y} 3 \mathrm{~m}-7 \mathrm{y} 6 \mathrm{~m}\end{array}$ & $\begin{array}{c}\quad(N=66) \\
\text { Mean }=5 \mathrm{y} 8 \mathrm{~m} \\
\text { Range }=4 \mathrm{y} 2 \mathrm{~m}-7 \mathrm{y} 7 \mathrm{~m}\end{array}$ \\
\hline
\end{tabular}


Table 2: Mean height (cm) for each drawing type for boys and girls

\begin{tabular}{|c|c|c|c|c|}
\hline & \multicolumn{3}{|c|}{ Drawing Type } & Grand Means \\
\hline Gender & Baseline & Positive & Negative & \\
\hline Boys & 14.08 & 18.70 & 16.42 & 16.40 \\
$(N=69)$ & $(\mathrm{SD}=7.04)$ & $(\mathrm{SD}=6.59)$ & $(\mathrm{SD}=7.33)$ & $(\mathrm{SD}=5.84)$ \\
\hline Girls & 14.04 & 15.87 & 13.86 & 14.59 \\
$($ N=63 $)$ & $(\mathrm{SD}=6.62)$ & $(\mathrm{SD}=7.02)$ & $(\mathrm{SD}=6.45)$ & $(\mathrm{SD}=5.94)$ \\
\hline Grand Mean & 14.06 & 17.35 & 15.20 & 15.54 \\
$($ N=132) & $(\mathrm{SD}=6.82)$ & $(\mathrm{SD}=6.92)$ & $(\mathrm{SD}=7.01)$ & $(\mathrm{SD}=5.94)$ \\
\hline
\end{tabular}


Table 3: Mean width (cm) for each drawing type for each age group

\begin{tabular}{|c|c|c|c|c|}
\hline Age Group & \multicolumn{3}{|c|}{ Drawing Type } & Grand \\
& \multicolumn{3}{|c|}{ Means } \\
\hline & Baseline & Positive & Negative & \\
\hline Youngest & 10.77 & 11.77 & 9.63 & 10.72 \\
$($ N=44 $)$ & $(\mathrm{SD}=5.37)$ & $(\mathrm{SD}=5.15)$ & $(\mathrm{SD}=5.00)$ & $(\mathrm{SD}=4.80)$ \\
\hline Middle & 8.45 & 9.52 & 8.70 & 8.89 \\
$($ N=44 $)$ & $(\mathrm{SD}=4.95)$ & $(\mathrm{SD}=3.94)$ & $(\mathrm{SD}=4.27)$ & $(\mathrm{SD}=3.61)$ \\
\hline Oldest & 8.30 & 10.57 & 10.45 & 9.77 \\
$($ N=44 $)$ & $(\mathrm{SD}=4.04)$ & $(\mathrm{SD}=4.93)$ & $(\mathrm{SD}=5.23)$ & $(\mathrm{SD}=4.03)$ \\
\hline Grand & 9.17 & 10.61 & 9.59 & 9.79 \\
Means & $(\mathrm{SD}=4.92)$ & $(\mathrm{SD}=4.76)$ & $(\mathrm{SD}=4.87)$ & $(\mathrm{SD}=4.85)$ \\
$($ N=132 $)$ & & & & \\
\hline
\end{tabular}


Table 4: Mean width (cm) for each drawing type for each group

\begin{tabular}{|c|c|c|c|c|}
\hline \multirow{2}{*}{ Group } & \multicolumn{3}{|c|}{ Drawing Type } & Grand Means \\
\hline & Baseline & Positive & Negative & \\
\hline SC & 6.59 & 8.94 & 7.29 & 7.61 \\
$(N=66)$ & $(\mathrm{SD}=3.10)$ & $(\mathrm{SD}=4.07)$ & $(\mathrm{SD}=3.80)$ & $(\mathrm{SD}=2.91)$ \\
\hline BC & 11.75 & 12.30 & 11.90 & 11.98 \\
$(N=66)$ & $(\mathrm{SD}=5.05)$ & $(\mathrm{SD}=4.83)$ & $(\mathrm{SD}=4.75)$ & $(\mathrm{SD}=4.19)$ \\
\hline Grand Means & 9.17 & 10.61 & 9.59 & 9.79 \\
$(N=132)$ & $(\mathrm{SD}=4.92)$ & $(\mathrm{SD}=4.76)$ & $(\mathrm{SD}=4.87)$ & $(\mathrm{SD}=4.85)$ \\
\hline
\end{tabular}


Table 5: Mean observed directional size use for each group

\begin{tabular}{|c|c|c|c|}
\hline Group & \multicolumn{2}{|c|}{ Drawing Type } & Grand Means \\
\hline & Positive & Negative & \\
\hline SC & 0.77 & 0.35 & 0.56 \\
$(N=66)$ & $(\mathrm{SD}=0.42)$ & $(\mathrm{SD}=0.48)$ & $(\mathrm{SD}=0.28)$ \\
\hline BC & 0.69 & 0.56 & 0.62 \\
$(N=66)$ & $(\mathrm{SD}=0.46)$ & $(\mathrm{SD}=0.50)$ & $(\mathrm{SD}=0.28)$ \\
\hline Grand Means & 0.73 & 0.45 & 0.59 \\
$(N=132)$ & $(\mathrm{SD}=0.44)$ & $(\mathrm{SD}=0.50)$ & $(\mathrm{SD}=0,28)$ \\
\hline
\end{tabular}


Table 6: Mean observed use of directional size change for boys and girls

\begin{tabular}{|c|c|c|c|}
\hline Gender & \multicolumn{2}{|c|}{ Drawing Type } & Grand Means \\
\hline & Positive & Negative & \\
\hline Boys & 0.80 & 0.36 & 0.59 \\
$(N=69)$ & $(\mathrm{SD}=0.39)$ & $(\mathrm{SD}=0.48)$ & $(\mathrm{SD}=0.26)$ \\
\hline Girls & 0.65 & 0.54 & 0.60 \\
$(N=63)$ & $(\mathrm{SD}=0.48)$ & $(\mathrm{SD}=0.50)$ & $(\mathrm{SD}=0.31)$ \\
\hline Grand Means & 0.73 & 0.45 & 0.59 \\
$(N=132)$ & $(\mathrm{SD}=0.44)$ & $(\mathrm{SD}=0.50)$ & $(\mathrm{SD}=0,28)$ \\
\hline
\end{tabular}


Table 7: Mean observed use of line quality for each drawing type for each group

\begin{tabular}{|c|c|c|c|}
\hline \multirow{2}{*}{ Group } & \multicolumn{2}{|c|}{ Drawing Type } & Grand Means \\
\hline SC & 0.12 & 0.47 & 0.30 \\
$(N=66)$ & $(\mathrm{SD}=0.33)$ & $(\mathrm{SD}=0.50)$ & $(\mathrm{SD}=0.32)$ \\
\hline BC & 0.23 & 0.35 & 0.29 \\
$(N=66)$ & $(\mathrm{SD}=0.42)$ & $(\mathrm{SD}=0.48)$ & $(\mathrm{SD}=0.35)$ \\
\hline Grand Means & 0.17 & 0.41 & 0.29 \\
$($ N=132) & $(\mathrm{SD}=0.38)$ & $(\mathrm{SD}=0.49)$ & $(\mathrm{SD}=0.33)$ \\
\hline
\end{tabular}

